\title{
Many-body phenomena in QED-cavity arrays
}

\author{
A. Tomadin ${ }^{1}$ and Rosario Fazio ${ }^{2}$ \\ ${ }^{1}$ Institute for Quantum Optics and Quantum Information of the Austrian Academy of Sciences, A-6020 Innsbruck, Austria \\ ${ }^{2}$ NEST, Scuola Normale Superiore \& INFM-CNR, Piazza dei Cavalieri 7, I-56126 Pisa, Italy
}

Coupled quantum electrodynamics (QED) cavities have been recently proposed as new systems to simulate a variety of equilibrium and non-equilibrium many-body phenomena. We present a brief review of their main properties together with a survey of the last developments of the field and some perspectives concerning their experimental realizations and possible new theoretical directions.

\section{INTRODUCTION}

The ability to design and fabricate controllable manybody systems has been realized to be a precious tool to explore the world of strongly correlated systems. In several different physical phenomena, ranging from high temperature superconductivity to heavy fermions or the fractional quantum Hall effect, strong local electronic correlations play a crucial role. The seemingly simplified models to describe these correlations, the Hubbard model of high temperature superconductivity for example, are extremely difficult to solve and over the years a number of judicious analytical and numerical methods have been developed. Together with these more "traditional" methods, in recent years it was proposed that strongly correlated systems could be studied by means of quantum simulators [1, i.e. fabricated systems that can experimentally simulate the model Hamiltonian underlying the non-trivial properties of the physical systems under consideration. The advantages of this approach are twofold. First of all it is possible to explore the properties of strongly correlated model Hamiltonians also in those regions of the phase diagram which are elusive to numerical and analytical investigations. Secondly it allows to test to which extent the model Hamiltonians under consideration are appropriate to treat the physical systems that they are supposed to describe or whether additional ingredients are necessary.

Quantum simulators have a long and successful story. Probably the first fabricated system to have these characteristics were Josephson junction arrays [2]. The field boosted with the appearance of cold atoms in optical lattices 3 which proved to be excellent simulators of a large variety of strongly interacting Fermi and Bose systems. Topic of the present paper is to review the latest developments of a newly born direction in the field of quantum simulators based on arrays of QED-cavities [4-6]. There is already a comprehensive review on the subject [7], in this paper we will focus on some of the recent developments.

Cavity-QED arrays offer the possibility to realize strongly correlated states of light. They can operate at high temperatures (as compared to Josephson arrays and optical lattices) and allow for single-site addressing thus opening a way to access experimentally correlation functions. Furthermore they might allow to explore a number of new equilibrium and non-equilibrium quantum phase transitions.

This paper is organized as follows. In the next section we will introduce cavity arrays. We define the model Hamiltonians that govern their dynamics and the most relevant sources of dissipation. In Sec. III we discuss the most important characteristics of the equilibrium phase diagram and the nature of the low-lying excitations. Section IV] describes how cavity arrays can operate as quantum simulators. Section $\mathrm{V}$ reviews some properties of these systems out of equilibrium. We complete the presentation with the conclusions where we will briefly discuss possible experimental realizations and some perspectives in the field.

\section{THE MODELS}

A sketch of a cavity array is illustrated in Fig. 1. It consists of a regular arrangement of QED-cavities which are coupled by the hopping of photons. Light resonates in each cavity and interacts with matter contained therein. Experimental realization of cavity arrays can be imagined in different ways. We will briefly discuss this point at the end of the paper. Fig. 1 illustrates the case in which the array is realized using photonic crystals. The salient ingredient at the base of the rich physics of cavity arrays is the interplay of two competing effects. Light-matter interaction inside the cavity leads to a (possibly strong) nonlinearity between photons. On the other hand, photon hopping between neighboring cavities favors delocalization thus competing with photon blockade. A model that describes a cavity array must then take into consideration the interaction of the light and the matter within each cavity, the coherent coupling between the cavities induced by the propagation of the light, the leakage of photons out of the cavities, and the dissipation and decoherence of the matter. It is also necessary to include an external pump to populate the cavities and devise a method to perform the measurement of the state of each cavity. We first describe the terms that lead to the unitary evolution of the array and afterwards the main sources of dissipation.

A single cavity confines several modes of the electromagnetic field and each mode is quantized as an harmonic oscillator. In the case that a single mode with frequency $\omega_{\mathrm{C}}$ is considered, the corresponding Hamiltonian is given by $\mathcal{H}_{\mathrm{C}}=\omega a_{\ell}^{\dagger} a_{\ell}$ where the operator $a_{\ell}\left(a_{\ell}^{\dagger}\right)$ annihilates 


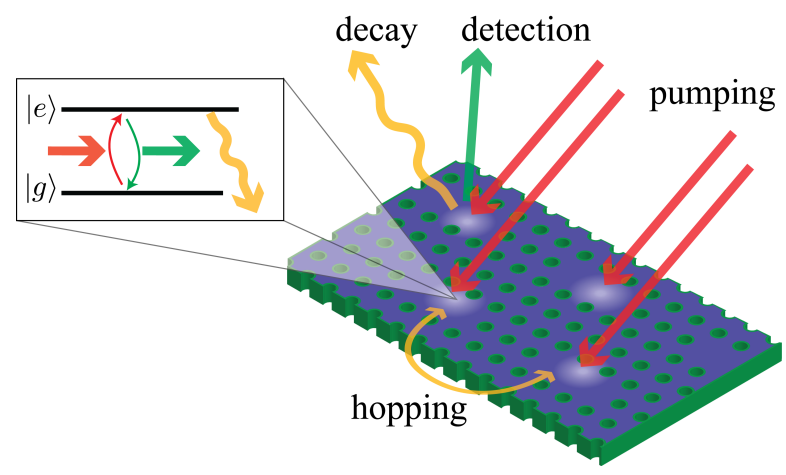

FIG. 1: A sketch of a QED-cavity array. It consists of a regular arrangement of QED cavities. Neighboring cavities are coupled by photon hopping. Nonlinearities in the cavities may produce an effective repulsion between the photons leading to an anharmonic spectrum. The nonlinearity may be produced, e.g. by a two-level system (depicted in the inset) coupled to the light resonating in the cavity and subjected to decay. Photons in the cavities have a finite lifetime therefore the cavities are pumped with an external coherent drive.

(creates) a quantum of light in the mode of the $\ell$-th cavity. If the cavities are sufficiently close to allow for photon hopping, an additional kinetic term $-J\left(a_{\ell^{\prime}}^{\dagger} a_{\ell}+\right.$ H.c. $)(J$ is associated to the tunneling rate) should be added to the Hamiltonian. These two terms constitute the model for a cavity array in the absence of any interaction with the matter field. In the presence of hopping the Hamiltonian of the photons is still harmonic and can be diagonalized by Fourier transform leading to the dispersion law of the photons in the lattice.

The presence of atoms (quantum dots or Cooper pair boxes depending on the implementation) inside each cavity leads to a strong effective nonlinearity between photons. It is enough to think to the matter field as a fewlevel system coupled to cavity mode and possibly to some external source. The Hamiltonian for an array of cavities can be written as on each cavity and the photon hopping term between different cavities

$$
\mathcal{H}=\sum_{\ell} \mathcal{H}_{\ell}^{(0)}-J \sum_{\left\langle\ell, \ell^{\prime}\right\rangle}\left(a_{\ell}^{\dagger} a_{\ell^{\prime}}+\text { H.c. }\right) \text {. }
$$

The local contribution $\mathcal{H}_{\ell}^{(0)}$ describes the light-matter interaction. In the limit in which leakage of photons is ignored, the Hamiltonian in Eq. (1) has been treated in the grand-canonical ensemble. In this case one should add a term containing the chemical potential. In the next section the phase diagram will be presented in the grand-canonical ensemble.

Probably the simplest model to describe the interaction between light and matter is the Jaynes-Cummings model in which one mode of the cavity interacts with a two-level system [8]. One photon can be absorbed by the two-level system, that goes into the excited state, and conversely a photon can be emitted if the two-level system flips from the excited $|2\rangle$ to the ground state $|1\rangle$.
The Jaynes-Cummings model reads

$$
\mathcal{H}_{\ell, \mathrm{JC}}^{(0)}=\varepsilon \sigma_{\ell}^{z}+\omega a_{\ell}^{\dagger} a_{\ell}+g\left(\sigma_{\ell}^{+} a_{\ell}+\sigma_{\ell}^{-} a_{\ell}^{\dagger}\right),
$$

$\sigma_{\ell}^{ \pm}$are the raising/lowering operators for the two-level system and $\varepsilon$ denotes the transition energy between the two levels. In the rotating frame with respect to the uncoupled Hamiltonian the relevant quantity is the detuning $\Delta=\omega-\varepsilon$. The spectrum of Eq. (2) is anharmonic so that, effectively, the two-level system induces a repulsion between the photons in the cavity. The strong effective nonlinearity between the photons turns the cavity into a turnstile device, where only a photon can be present at the same time. Intuitively, this can be understood as the fact that one photon in the cavity strongly modifies the effective resonance frequency, inhibiting the injection of a second photon. This phenomenon has been termed "photon blockade" 9], after the Coulomb blockade effect of electrons in mesoscopic structures. The entire cavity thus behaves as an effective spin system, that emits strongly anti-bunched light. The cavity array Hamiltonian with the Jaynes-Cummings term was first used by Greentree et al. in Ref. [5] and by Angelakis et al. in Ref. 6]. A similar model with many two-level systems in each cavity has also been used [10 12, where each twolevel system interacts with the mode of the cavity in a sort of Dicke-Bose-Hubbard model. Na et al. in Ref. [1] allowed for the number of systems to fluctuate among the cavities and showed that if the cavity is strongly reddetuned with respect to the transition in the two-level systems, the photons experience an effective Kerr nonlinearity. Koch and Le Hur [13, though, pointed out that the substitution of the Jaynes-Cummings coupling with an effective Kerr nonlinearity is not appropriate in general.

Since the quantum effects in the cavity may be visible if the coupling exceeds the decay amplitude of the matter and of the light, there has been a lot of effort in increasing the effective cavity nonlinearity. Strong interaction of light is in general limited by the absorption of the medium, because the optical nonlinearities are weak in non-resonant processes, while the absorption plays a dominant role in the resonant processes. One route to access the strong-coupling regime involves the usage of external classical sources that prepare coherently the matter in a state with reduced absorption. Electromagnetically Induced Transparency (EIT) can be used to achieve interaction strengths that are one order of magnitude larger than what is possible with an ensemble of two-level systems [14. Various schemes to obtain strong nonlinearities have been discussed [9, 15] 17]. In this spirit Hartmann et al. in Ref. 4] considered an ensemble of four-level atoms in a cavity array defined, in the rotating frame, by a local interaction of the form

$$
\begin{aligned}
\mathcal{H}_{\ell, \mathrm{EIT}}^{(0)} & =\delta S_{\ell}^{33}+\Delta S_{\ell}^{44}+\Omega\left(S_{\ell}^{23}+S_{\ell}^{32}\right) \\
& +g_{1}\left(S_{\ell}^{13} a_{\ell}^{\dagger}+S_{\ell}^{31} a_{\ell}\right)+g_{2}\left(S_{\ell}^{24} a_{\ell}^{\dagger}+S_{\ell}^{42} a_{\ell}\right),
\end{aligned}
$$

having defined the global atomic raising and lowering 
operators $S^{l m}=\sum_{j=1}^{N}|l\rangle_{j}\left\langle m\left|, \sigma_{j}^{l m}=\right| l\right\rangle_{j}\langle m|$ are the atomic raising and lowering operators $(l \neq m)$, or energy level populations $(l=m)$ for the $j$ th atom. The transition $|3\rangle_{j} \rightarrow|2\rangle_{j}$ is driven by a classical coupling field with Rabi frequency $\Omega$; the cavity mode couples the $|1\rangle_{j} \rightarrow|3\rangle_{j}$ and $|2\rangle_{j} \rightarrow|4\rangle_{j}$ transitions with coupling constants $g_{1}$ and $g_{2}$; the parameters $\delta$ and $\Delta$ account for the detunings of levels 3 and 4, respectively. They considered a setup similar to Ref. [9] and introduced three families of polaritons, delocalized over the whole atomic ensemble, that diagonalize the Hamiltonian (if the state $|4\rangle$ decouples from the dynamics and the number $N$ of atoms is very large). One of the polaritons projects only on the lowest-lying metastable states $|1\rangle$ and $|2\rangle$ and is thus the longest-lived. The polaritons can be considered independent bosonic particles if their number is much smaller than the number of atoms inside each cavity [18. The longest-lived polariton decouples from the others and experiences an energy shift due to the perturbative coupling to the level $|4\rangle$, that induces an interaction of the Kerr form. In this way, in the large $N$ limit, they were able to show that the cavity model maps onto an effective Bose-Hubbard model [19] for polaritons

$$
\mathcal{H}_{\mathrm{BH}}=-J \sum_{\left\langle\ell \ell^{\prime}\right\rangle} a_{\ell^{\prime}}^{\dagger} a_{\ell}+\frac{U}{2} \sum_{\ell} a_{\ell}^{\dagger} a_{\ell}^{\dagger} a_{\ell} a_{\ell} .
$$

Up to now we did not take into account the leakage of photons out of the cavities and the decoherence and dissipation of the few-level systems. One possibility, in the case of slow decay, is to complement the Hamiltonian with imaginary frequencies multiplied by the projectors of the decaying states, that render the dynamics non-Hermitian (see some application of this method in Ref. [14]). Alternatively the decay can be given in terms of the density matrix $\rho$ of the cavity array, using the master equation

$$
\partial_{t} \rho(t)=-i[\mathcal{H}, \rho(t)]+\mathcal{L}[\rho(t)],
$$

where the Liouvillian $\mathcal{L}$ in the Lindblad form reads e.g.

$$
\mathcal{L}[\rho(t)]=\frac{\kappa}{2} \sum_{\ell}\left(a_{\ell} \rho(t) a_{\ell}^{\dagger}-a_{\ell}^{\dagger} a_{\ell} \rho(t)-\rho(t) a_{\ell}^{\dagger} a_{\ell}\right) .
$$

This term describes leakage of photons from all the cavities of the array, with an equal rate $\kappa$. A similar term can be used to describe decoherence and dissipation of the matter, although these processes can usually be neglected in the timescale of the photonic decay 7 .

In order to have a measurable signal out of the cavity array it is necessary to refill with photons the modes of the cavities, to contrast the leakage. If a coherent laser beam of frequency $\omega_{\mathrm{L}}$ is coupled to the cavity, the Hamiltonian acquires a term $\varepsilon(t)_{L} a_{\ell}^{\dagger}+\varepsilon(t)_{L}^{*} a_{\ell}$, where $\varepsilon(t)_{L}$ is proportional to the electric field of the beam. To eliminate the explicit time-dependence $e^{i \omega_{\mathrm{L}} t}$ from $\varepsilon(t)_{L}$ it is convenient to use a rotating frame in which the strength of the pumping is constant and the frequency $\omega_{L}$ is subtracted from the energy of the photons in the cavity mode. The contribution of the drive to the Hamiltonian reads

$$
\mathcal{H}_{\ell, \mathrm{D}}=-\omega_{\mathrm{L}} a_{\ell}^{\dagger} a_{\ell}+\varepsilon_{L} a_{\ell}^{\dagger}+\varepsilon_{L}^{*} a_{\ell} .
$$

In the next section we will discuss the main features of the phase diagram deriving from the Hamiltonian (1).

\section{PHASE DIAGRAM}

The phase diagram of the Bose-Hubbard model defined in Eq. (4) has been studied for more than two decades (see for example references in [3]). A qualitative understanding of the zero-temperature phase diagram can be obtained by considering the two limiting cases in which one of the two coupling energies $(J$ or $U$ ) dominates. If the hopping dominates, the bosons delocalize throughout the whole lattice. In the limit of vanishing interactions, the many-body ground state becomes simply an ideal Bose-Einstein condensate where all the bosonic particles are in the Bloch state of the lattice with vanishing quasimomentum, so that the many-body wavefunction is written as $\left(\frac{1}{\sqrt{L}} \sum_{\ell=1}^{L} a_{\ell}^{\dagger}\right)^{N} \mid$ vac $\rangle$, where $L$ is the total number of cavities, $N$ is the total number of bosons and $|v a c\rangle$ is the vacuum state of the many-body Fock basis. In contrast, if the interaction dominates, each site has a well defined number of bosons in the ground state. In order to put an extra boson on a given site, one has to overcome an energy gap of the order of $U$. In the Mott lobes each cavity contains a definite number of bosons, the fluctuations of the number operator vanish, and each particle is localized in a given cavity. Each Mott lobe exists up to a maximum hopping $(J / U)_{\text {crit }}$ at which it is energetically convenient for the particles to delocalize over the whole lattice (decreasing their kinetic energy) rather than localize in a given site (decreasing their mutual interaction). In the case of vanishing hopping, the Hamiltonian is the sum of commuting local Hamiltonians and the ground state can be written in the product form $\prod_{\ell}|\bar{n}\rangle_{\ell}$. When the hopping is comparable with the onsite repulsion a quantum phase transition takes place, separating a superfluid from a Mott insulator. Although there are large quantitative differences between the case of one-, two-, or three-dimensional lattices, the equilibrium phase diagram of the model (at zero temperature, in the plane of the hopping and the chemical potential) is similar in all cases and consists of a superfluid phase surrounding a sequence of Mott-insulator lobes [see Fig. 2 (left panel)]. The $U(1)$ symmetry is broken in the superfluid phase, where the kinetic energy dominates.

The phase diagram of the cavity array described by Eq. (1) resembles strong similarities with that of the Bose-Hubbard model. In Fig. 2 (right panel) we report for comparison the phase diagram obtained in Ref. [10] for a one-dimensional array. Quantitative differences 

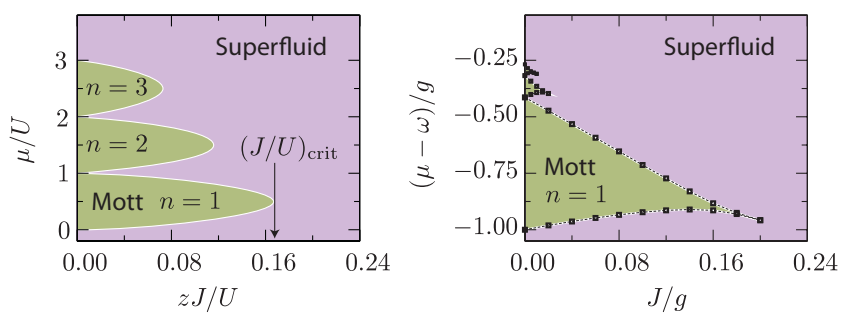

FIG. 2: Left: sketch of the phase diagram of the BH model (4). The green regions are the Mott lobes, $z$ is the coordination number of the lattice. Each lobe extends up to a maximum critical ratio $(J / U)_{\text {crit }}$. Right: the phase diagram of the cavity array in the case of a Jaynes-Cummings model, according to the DMRG computation presented in Ref. [10]. The green area corresponds to a Mott insulator of polaritons with average filling $n=1,2$, and 3 .

arise depending on the model of the cavity. For the sake of clarity we will ignore these differences and try to highlight only those characteristics which are common to cavity arrays. The phase transition between the Mottinsulator and the superfluid is a symmetry breaking phenomenon that involves the conservation of the number of bosons $\sum_{\ell} n_{\ell}$. The conserved quantity in the cavity model, take for example the Jaynes-Cummings interaction, instead is the number of polaritons, that do not have a definite quantum statistics. In cavity arrays the detuning $\Delta$ can also be varied so that the phase diagram becomes effectively three-dimensional [13]. It turns out that the detuning is a convenient experimental knob to tune the system across the phase transition. Moreover, most importantly, to change the detuning from negative to positive values drives the nature of the excitations from quasilocalized excitons to polaritons to weakly interacting photons 20].

A cavity array exhibits spectral properties similar to the BH models, including gapped particle and hole bands in the Mott insulating phase and Bogoliubov-type excitations in the superfluid phase. The single-particle excitation spectrum in the Mott phase has been investigated also by Aichorn et al. 20] using a variational cluster approach, in which the self-energy of the infinite lattice of cavities is approximated by a finite reference system. Schmidt and Blatter 21, for the Jaynes-Cummings case, found four modes corresponding to particle or hole excitations, versus the two modes of the BH model. Pippan et al. in Ref. 22 studied the dynamical structure factor and the single-particle spectrum using a quantum Monte Carlo simulation in one dimension. An analysis of the nature of the excitations in small clusters was also performed in Ref. 23]. Paternostro et al. in Ref. 24] investigated the dynamical properties of an array with many two-level systems in each cavity, beyond the elementary excitation picture and found that the time-evolution of the array, in the mean-field approximation, supports a soliton-like excitation.

Following the initial investigations [4] $[$, the existence of the Mott lobes in the cavity arrays has been verified with several different methods. Makin et al. 25] resorted to the exact diagonalization of small sets of cavities with several topologies and to a cluster-mean-field approximation. Schmidt et al. in Ref. 21] proposed a strongcoupling theory based on a linked-cluster expansion of the Green's functions at finite temperature. This method includes quantum fluctuations beyond the mean-field results, that are recovered instead in the random-phase approximation. With these spectral methods the border of the Mott lobe is signaled by the vanishing of the gap. A quantum Monte Carlo analysis in two dimensions has been performed by Zhao et al. in Ref. [26]. The quantum Monte Carlo results for the phase border compare quantitatively with the analytical solution 21 almost up to the tip of the Mott lobe. In one dimension, the existence of the insulating Mott lobes has been established in Ref. 10 by means of a DMRG calculation. With the same method, it has been shown in Ref. [27] that the polariton fluctuations in a finite block of cavities yields a clear signature of the two phases. The universality class of the transition appears of the same type as the $\mathrm{BH}$ [21, 22].

The nature of the Mott-insulator for polaritons is different from the $\mathrm{BH}$ case, in that the number of polaritons is fixed to an integer value, but the fluctuations of the number of photons $\left\langle n_{\ell}\right\rangle$ do not vanish. Similarly, in Ref. 22] it was shown that the exciton and photon structure factors cannot be used to characterize the Mott state. Although the photons are not the genuine excitation of the system, it is desirable to characterize the transition in terms of photonic observables since the emission of light from the cavity can be measured in experiments. To measure the polaritonic state using the projection on the atomic component [4, 6] it is necessary to address the system with further optical probes.

The presence of many two-level systems in each cavity and its effect on the phase diagram has been analyzed in 10, 11, confirming that even with a moderate number of atoms in each cavity there is a good quantitative agreement with the mapping onto the $\mathrm{BH}$ performed in [4. New features appear if the number of atoms fluctuates from cavity to cavity. In this case a glassy phase for polaritons is expected around the Mott lobes [10. Also disorder in the distribution of the excitation and cavity frequencies 11, and the effects of a finite temperature [20, 25] have been considered.

Using more complex models of cavity arrays a twocomponent Bose-Hubbard model 28] and superradiant Mott phase 29] have been shown to be realizable.

\section{CAVITY ARRAYS AS QUANTUM SIMULATORS}

Building upon the models presented in Sec. II] in specific parameter ranges or with the addition of further optical tools, it is possible to use the cavity array as an 
implementation of other many-body models. In other words, the cavity array offers an implementation of a "quantum simulator" for lattice models, in the same spirit as the Josephson junctions arrays or the cold atoms in optical lattices. The first requirement for a quantum simulator is to act as a calculator specifically tailored to the solution of the model that it implements. The knowledge on the model gained with the quantum simulator can then be applied to all the other physical systems described by the the same model. With respect to the implementation of a quantum simulator in an optical lattice, the cavity array may offer the advantage that each site of the array can be addressed independently. The expectation values of the observables could then be measured directly from the light emitted by one cavity. Moreover, the cavity arrays can be implemented with several different experimental systems and this may offer some advantages. On the other side, optical lattices seem unbeatable in terms of scalability and absence of imperfections. It should be said that at present there are yet no experimental realizations of cavity arrays. The hypothetical advantages of these quantum simulators need to be tested against experimental realizations.

The earliest family of lattice many-body systems that have been investigated in the context of cavity arrays is spin lattice systems. The indication that a single cavity could act as an effective spin system for the photons dates back to the recognition of the photon blockade effect [9]. Angelakis et al. [6] derived an effective XY Hamiltonian from the Jaynes-Cummings model after decoupling the upper and the lower polariton in the Mott polaritonic phase. The realization of a ZZ Ising coupling $\sigma_{\ell}^{z} \sigma_{\ell+1}^{z}$ has also been discussed [30] and the possibility to achieve both XY and ZZ couplings has been put forward by Hartmann et al. in Ref. 31. The proposal relies on a cavity array containing three-level atoms, in a $\Lambda$ configuration. The two lowest-lying states $|1\rangle$ and $|2\rangle$ are coupled to the excited state $|3\rangle$ both via the quantized mode of the cavity and via external classical sources. With an appropriate choice of the couplings and the detunings, the dominant Raman transition between the two lowest-lying states involves one laser and one cavity photon. This implies that the emission and absorption of virtual photons in the cavity is accompanied by a transition between the two lowest-lying states, that become the effective $1 / 2$ spin in the cavity. The coupling of the virtual photons between neighboring cavities produces a XY model. This effective spin can be coupled to an effective magnetic field in the $z$ direction. With the same atomic spectrum but a different configuration of the external sources, it is possible to implement a ZZ interaction of the form $\sigma_{\ell}^{z} \sigma_{\ell+1}^{z}$. The XY and the ZZ couplings do not act at the same time, but are combined in a unique effective Hamiltonian with the application of the Suzuki-Trotter decomposition in which the lasers that produce either interaction are periodically flashed one after the other. Cho et al. 32 focused on the implementation of high-spin Heisenberg models. Given the lack of analytical or numerical infor- mations that are available today on the phase diagram of high-spin systems, the possibility to measure the phase diagram directly from a quantum simulator is very interesting. The scheme includes an ensemble of threelevel atoms in a configuration similar to Ref. 31, but relies on fixed external lasers. The model allows to simulate terms in the Hamiltonian of the form $S_{\ell}^{2},\left(S_{\ell}^{z}\right)^{2}, S_{\ell}^{z}$, $S_{\ell}^{z} S_{\ell+1}^{z}, S_{\ell}^{x} S_{\ell+1}^{x}, S_{\ell}^{y} S_{\ell+1}^{y}$, and $S_{\ell}^{z} S_{\ell+1}^{z}$. Quite a few external sources are necessary to control independently all the constants in the model, while the magnitude of the spin is determined by the number of two-level systems in the cavity.

Taking into account the polarization of light in the microcavities, Ji et al. in Ref. 33. put forward a model that is able to exhibit ferromagnetism of the photonic circular polarization. The model considers an hexagonal photonic crystal with a square superlattice of band gap cavities, doped with three-level systems. In the same system, it is also possible to access adiabatically the antiferromagnetic phase, and an exotic super-counter-fluid phase in which the total current of photons is zero as in the Mott phase, but the currents with definite circular polarization are nonzero and opposite.

The local addressability of cavity arrays has been exploited by Cho et al. in Ref. 34 to propose the simulation of hard-core bosons with Abelian vector potentials. In particular this method allows the simulation of the Laughlin wave function for the fractional quantum Hall effect. The proposal assumes a two-dimensional array of cavities, each containing a single three-level system. To address independently the two directions of the array, two modes of each resonator are used, with the assumption that the frequency difference between the two modes is much larger than the coupling strength $g$. The simulation of the hard-core bosons follows from the simulation of a spin system using the lowest-lying states of the $\Lambda$ configuration, along the lines of Ref. 32. The amplitude of the interaction between the spins on neighboring sites is proportional to a position-dependent phase, that defines the gauge potential. This phase is controlled by the external laser sources acting on the three-level system and the local addressability of the cavities is invaluable to the precise implementation of this phase. Moreover, by an adiabatic change of the laser phases, it is possible to insert a flux quantum through the two-dimensional plane, thus creating and moving a quasi-excitation in the system.

Cavity arrays can be used also to store and manipulate the resources necessary to perform quantum computation. The first study in this direction has been made by Angelakis et al. in Ref. [35] with the aim to realize quantum gates with photonic-crystal waveguides. In this case the coupling between the photons and the matter is not present in each cavity, but is used only in some selected points of the quantum circuit to induce a phase gate between the qubits. The coherent control of the photonic transmission through a cavity array has been considered by $\mathrm{Hu}$ et al. in Ref. [36, where it was 


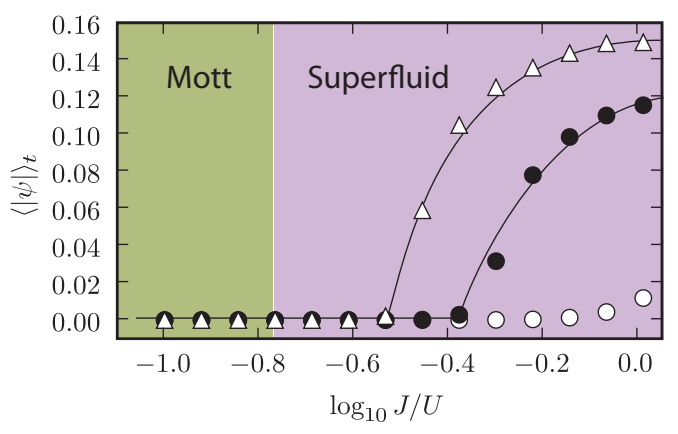

FIG. 3: Non-equilibrium signature of the quantum phase transition between the Mott-insulator and the superfluid state in the Bose-Hubbard model (4) with the leakage of photons described by (6), obtained by Tomadin et al. in Ref. 40. The order parameter $\psi=\langle a\rangle$, averaged in an interval of time, is shown for $U / \kappa=50$ (empty circles), 100 (filled circles), and 200 (empty triangles). The transition of the order parameter takes place together with a transition from antibunched to bunched light. On decreasing the dissipation strength the onset at which the order parameter becomes non-zero approaches the equilibrium value (indicated as a vertical line).

shown, by a Green's function technique, that the dispersion of the hybrid light-matter excitations in the array can be controlled by acting on the population inversion of the dopants. A procedure to generate indistinguishable single photons or polarization-entangled photonic pairs, which are important resources to the implementation of the quantum computation algorithms, has been demonstrated in Ref. [37] by Na et al. resorting to the exact integration of a small number of cavities. The complete implementation of the Grover's search algorithm has been proposed recently by Kyoseva et al. 38] together with a proposal to implement one-way quantum computation with cavity arrays [39].

\section{NON-EQUILIBRIUM BEHAVIOR}

In order to detect experimentally the different quantum phases presented in Sec. III one assumes that the mixed light-matter excitations have a lifetime that is longer than the time necessary to the measurement process. Similarly, in order to perform the quantum simulations of a ground state discussed in Sec. IV] it is necessary that the timescale of the many-body dynamics is shorter than the decay time. For example, the ground state of a many-body system could be reached if the decay of the polaritons is at least slower than the intercavity tunneling [11. As the decay times of the photons and of the matter strongly depend on the specific implementation [7], it is interesting to consider schemes that allow to measure the signatures of the many-body physics without relying on a negligible dissipation. It is then necessary to study the cavity array under strong non-equilibrium conditions.

Carusotto et al. in Ref. 41] investigated a system of photons in a circular array of cavities in the TonksGirardeau limit. All the cavities are driven by a single laser beam and the far-field emission, that gives access to the occupation of the single-particle states in the momentum representation, is computed. The laser couples to the many-body wavefunctions supported by the array depending on the total energy of the state. This yields a spectroscopic analysis of a many-body system that is created by the probe beam itself. In the limit of very strong Kerr nonlinearity, the photons become hard-core bosons and, being in one dimension, are represented by an effective wave function for noninteracting fermions. The classification of the states given by this mapping is used also to characterize the absorption in the case of intermediate interaction strength.

The quantum phase transition of the $\mathrm{BH}$ (see Sec. III), in an open cavity array, has been investigated by Tomadin et al. in Ref. [40] who proposed to discriminate the Mott-insulator and the superfluid phases analyzing the light emitted by the cavity array following a pulse that creates a Mott state in the system. The existence of the two phases is clearly seen in the coherence properties of the emitted light (see Fig. 3) for currently achievable values of the ratio between the interaction and the dissipation. The method relies on the dynamical instability of the $\mathrm{BH}$ that follows a quantum quench from the Mott to the superfluid phase. The realization of the quantum quench in the dissipative environment is realized with a careful design of the initial pulse, that is substantially different from a $\pi$-pulse, and it is shown that the detection of the phase border is very robust to imperfections in the pumping. The time-evolution of the system produces antibunched light if the ratio $J / U$ is smaller than the critical value $(J / U)_{\text {crit }}$ at the tip of the Mott lobe, and the crossover from antibunched to bunched light clearly marks the phase boundary.

\section{CONCLUSIONS}

As candidates for simulating strongly interacting models, coupled cavities present new characteristics as compared to other successful examples, like optical lattices or Josephson junction arrays: most notably it is possible to access their local properties. In this paper we briefly review their basic properties and some of the latest developments in the field.

QED-cavities can be realized in a number of different ways 42 44. This flexibility in the design is a potential advantage to realize different local nonlinearities. Moreover different systems may allow for different measurement schemes as well. As already mentioned, at present there are no experimental realizations of cavity arrays although it seems that technological requirements are already at hand to fabricate a small number of coupled cavities. In this respect it may be interesting to explore properties of small clusters.

In this review we confined the discussion to coupled 
cavities. There are other systems in which many-body physics with light can be realized (see for example [45, 46]). Due to space limitations we did not touch these very interesting directions of research.

\section{Acknowledgments}

We acknowledge a very fruitful collaboration with Iacopo Carusotto, Dario Gerace, Vittorio Giovannetti,
Atac Imamoglu, Davide Rossini, Giuseppe Santoro, and Hakan Tureci. We benefitted from many discussions with Dimitris Angelakis, Michael Hartmann, and Martin Plenio. The work was supported by EU project IP-Eurosqip.
[1] E. Jané, G. Vidal, W. Dür, P. Zoller, and J. I. Cirac, "Simulation of quantum dynamics with quantum optical systems," Quantum Inf. and Comp. 3, 15-37 (2003).

[2] R. Fazio and H. S. J. van der Zant, "Quantum Phase Transitions and Vortex Dynamics in Superconducting Networks," Phys. Rep. 355, 235-334 (2001).

[3] M. Lewenstein, A. Sanpera, V. Ahufinger, B. Damski, A. Sen (De), and U. Sen, "Ultracold atomic gases in optical lattices: Mimicking condensed matter physics and beyond," Adv. Phys. 56, 243-279 (2007).

[4] M. J. Hartmann, F. G. S. L. Brandao, and M. B. Plenio, "Strongly interacting polaritons in coupled arrays of cavities," Nature Phys. 2, 849-855 (2006).

[5] A. D. Greentree, C. Tahan, J. H. Cole, and C. L. Hollenberg, "Quantum phase transitions of light," Nature Phys. 2, 856-861 (2006).

[6] D. G. Angelakis, M. F. Santos, and S. Bose, "Photonblockade-induced Mott transitions and $X Y$ spin models in coupled cavity arrays," Phys. Rev. A 76, 031805(R) (2007).

[7] M. J. Hartmann, F. G. S. L. Brandao, and M. P. Plenio, "Quantum many-body phenomena in coupled cavity arrays," Laser \& Photon. Rev. 2, 527-556 (2008).

[8] B. W. Shore and P. L. Knight, "The Jaynes-Cummings Model," J. Mod. Opt. 40, 1195-1238 (1993).

[9] A. Imamoglu, H. Schmidt, G. Woods, and M. Deutsch, "Strongly interacting photons in a nonlinear cavity," Phys. Rev. Lett. 79, 1467-1470 (1997).

[10] D. Rossini and R. Fazio, "Mott-insulating and glassy phases of polaritons in 1D arrays of coupled cavities," Phys. Rev. Lett. 99, 186401 (2007).

[11] N. Na, S. Utsunomiya, L. Tian, and Y. Yamamoto, "Strongly correlated polaritons in a two-dimensional array of photonic crystal microcavities," Phys. Rev. A 77, 031803(R) (2008).

[12] S.-C. Lei and R.-K. Lee, "Quantum phase transitions of light in the Dicke-Bose-Hubbard model," Phys. Rev. A 77, 033827 (2008).

[13] J. Koch and K. Le Hur, "Superfluid-Mott insulator transition of light in the Jaynes-Cummings lattice," Phys. Rev. A 80, 023811 (2009).

[14] M. Fleischhauer, A. Imamoglu, and J. P. Marangos, "Electromagnetically induced transparency: Optics in coherent media," Rev. Mod. Phys. 77, 633-673 (2005).

[15] H. Schmidt and A. Imamoglu, "Giant Kerr nonlinearities obtained by electromagnetically induced transparency," Opt. Lett. 21, 1936-1938 (1996).

[16] M. J. Hartmann and M. B. Plenio, "Strong photon non- linearities and photonic Mott insulators," Phys. Rev. Lett. 99, 103601 (2007).

[17] F. G. S. L. Brandao, M. J. Hartmann, and M. B. Plenio, "Light-shift-induced photonic nonlinearities," New J. Phys. 10, 043010 (2008).

[18] The notation $a_{\ell}$ is used to denote exact (photons) and approximated (polaritons) bosonic particles.

[19] M. P. Fisher, P. B. Weichmann, G. Grinstein, and D. S. Fisher, "Boson localization and the superfluid-insulator transition," Phys. Rev. B 40, 546-570 (1989).

[20] M. Aichorn, M. Hohenadler, C. Tahan, and P. B. Littlewood, "Quantum fluctuations, temperature, and detuning effects in solid-light systems," Phys. Rev. Lett. 100, 216401 (2008).

[21] S. Schmidt and G. Blatter, "Strong coupling theory for the Jaynes-Cummings-Hubbard model," Phys. Rev. Lett. 103, 086403 (2009).

[22] P. Pippan, H. G. Evertz, and M. Hohenadler, "Excitation spectra of strongly correlated lattice bosons and polaritons," Phys. Rev. Lett. 80, 033612 (2009).

[23] E. K. Irish, C. D. Ogden, and M. S. Kim, "Polaritonic characteristics of insulator and superfluid states in a coupled-cavity array," Phys. Rev. A 77, 033801 (2008).

[24] M. Paternostro, G. S. Agarwal, and M. S. Kim, "Solitonic behaviour in coupled multi atom-cavity systems," New Jour. Phys. 11, 013059 (2009).

[25] M. I. Makin, J. H. Cole, C. Tahan, L. C. L. Hollenberg, and A. D. Greentree, "Quantum phase transitions in photonic cavities with two-level systems," Phys. Rev. A 77, 053819 (2008).

[26] J. Zhao, A. W. Sandvik, and K. Ueda, "Insulator to superfluid transition in coupled photonic cavities in two dimensions," arXiv:0806.3603

[27] D. Rossini, R. Fazio, and G.E. Santoro, "Photon and polariton fluctuations in arrays of QED-cavities," Europhys. Lett. 83, 47001 (2008).

[28] M. J. Hartmann, F. G. S. L. Brandao, and M. B. Plenio, "A polaritonic two-component Bose-Hubbard model," New J. Phys. 10, 033011 (2008).

[29] M. J. Bhaseen, M. Hohenadler, A. O. Silver, and B. D. Simons, "Polaritons and Pairing Phenomena in Bose-Hubbard Mixtures," Phys. Rev. Lett. 102, 135301 (2009).

[30] P. B. Li, Y. Gu, Q. H. Gong, and G. C. Guo, "Generation of Ising interaction and cluster states in a onedimensional coupled resonator waveguide," E. Phys. J. D 55, 205-209 (2009).

[31] M. J. Hartmann, F. G. S. L. Brandao, and M. B. Plenio, 
"Effective spin systems in coupled microcavities," Phys. Rev. Lett. 99, 160501 (2007).

[32] J. Cho, D. G. Angelakis, and S. Bose, "Heralded generation of entanglement with coupled cavities," Phys. Rev. A 78, 022323 (2008).

[33] A.-C. Ji, X. C. Xie, and W. M. Liu, "Quantum magnetic dynamics of polarized light in arrays of microcavities," Phys. Rev. Lett. 99, 183602 (2007).

[34] J. Cho, D. G. Angelakis, and S. Bose, "Fractional quantum Hall state in coupled cavities," Phys. Rev. Lett. 101, 246809 (2008).

[35] D. G. Angelakis, M. F. Santos, V. Yannopapas, and A. Ekert, "A proposal for the implementation of quantum gates with photonic-crystal waveguides," Phys. Lett. A 362, 377-380 (2007).

[36] F. M. Hu, L. Zhou, T. Shi, and C. P. Sun, "Coupled cavity QED for coherent control of photon transmission: Green-function approach for hybrid systems with twolevel doping," Phys. Rev. A 76, 013819 (2007).

[37] N. Na and Y. Yamamoto, "Generation of indistinguishable single photons and polarization-entangled photonpairs via polaritonic superfluid to Mott-insulator quantum phase transition," arXiv:0804.1829.

[38] E. S. Kyoseva, D. G. Angelakis, and L. C. Kwek, "A single-interaction step implementation of a quantum search in coupled micro-cavities," arXiv:0908.3308.

[39] A. Kay and D. G. Angelakis, "Reproducing spin lattice models in strongly coupled atom-cavity systems," Europhys. Lett. 84, 20001 (2008).
[40] A. Tomadin, V. Giovannetti, R. Fazio, D. Gerace, I. Carusotto, H. E. Tureci, and A. Imamoglu, "Nonequilibrium phase transition in driven-dissipative nonlinear cavity arrays," arXiv:0904.4437.

[41] I. Carusotto, D. Gerace, H. E. Tureci, S. De Liberato, C. Ciuti, and A. Imamoglu, "Fermionized photons in an array of driven dissipative nonlinear cavities," Phys. Rev. Lett. 103, 033601 (2009).

[42] A. Wallraff, D. Schuster, A. Blais, L. Frunzio, R. Huang, J. Majer, S. Kumar, S. Girvin, and R. Schoelkopf, "Strong coupling of a single photon to a superconducting qubit using circuit quantum electrodynamics," Nature 431, 162-167 (2004).

[43] K. Hennessy, A. Badolato, M. Winger, D. Gerace, M. Atature, S. Gulde, S. Falt, E. L. Hu, and A. Imamoglu, "Quantum nature of a strongly coupled single quantum dotcavity system," Nature 445, 896-899 (2007).

[44] A. Faraon, I. Fushman, D. Englund, N. Stoltz, P. Petroff, and J. Vuckovic, "Coherent generation of non-classical light on a chip via photon-induced tunnelling and blockade," Nature Phys. 4, 859-863 (2008).

[45] D. E. Chang, V. Gritsev, G. Morigi, V. Vuletic, M. D. Lukin, and E. A. Demler, "Crystallization of strongly interacting photons in a nonlinear optical fibre," Nature Phys. 4, 884-889 (2008).

[46] M. Kiffner and M. J. Hartmann, "Dissipation induced Tonks-Girardeau gas of photons," arXiv:0908.2055. 